\title{
19. Pädiatrischer Frühling
}

Gespräch mit emer. Univ.-Prof. Dr. Wilhelm Müller, der gemeinsam mit Univ.-Prof. Dr. Reinhold Kerbl die wissenschaftliche Leitung des Pädiatrischen Frühlings, 23.-25. Mai 2019, innehat.

Pädiatrie \& Pädologie: Was ist Ihrer Meinung nach das Geheimnis des Erfolgs dieser Veranstaltung?

Müller: Ich denke, es ist die Mischung aus Fachvorträgen, Workshops und Umfeld. Wir bemühen uns einerseits um hochqualitative Fortbildung mit Berücksichtigung aktueller Entwicklungen. Gleichzeitig ist es uns wichtig, dass sich unser Publikum bei uns auch wohlfühlt. Jahreszeit und Gegend tragen dazu schon wesentlich bei. Und in der Programmgestaltung achten wir sehr darauf, dass auch die persönliche Kommunikation nicht zu kurz kommt. Weil Meinungs- und Erfahrungsaustausch wichtige Bestandteile guter Fortbildung sind.

Hat die Veranstaltung ein Generalthema?

Müller: Wie in den letzten Jahren haben wir auf ein eigentliches Generalthema verzichtet. Weil die Pädiatrie so vielseitig ist, dass jegliches Generalthema eine unnötige Einschränkung darstellen würde. Wir möchten stattdessen in möglichst vielen Bereichen der Pädiatrie aktuelle und praxisbezogene Fortbildung vermitteln. Mit den für heuer gewonnenen Referentinnen und Referenten ist dies meine Erachtens auch wieder gewährleistet.

Gibt es heuer einen Vortrag oder einen Workshop, den Sie besonders hervorheben möchten?

Müller: Wir bemühen uns jedes Jahr, wieder "etwas Neues" anzubieten. Heuer möchte ich - ohne andere zurückstellen zu wollen den erstmals angebotenen Work- shop „Jugendmedizin“ erwähnen. Ich glaube dass die Pädiatrie da Defizite hat, die es auszugleichen gilt. Großen Anklang findet auch der Workshop „Entwicklungsdiagnostik", bei dem auch eine neue Praxis-App vorgestellt wird. Für die Plenarvorträge freuen wir uns wieder auf die pädiatrisch-dermatologischen Beiträge von Herrn Professor Höger, neu sind diesmal u.a. die Referate über "Jungenmedizin“ und "Suchterkrankungen“.

Gibt es Neuerungen beim Pädiatrischen Frühling 2019, und welche?

Müller: Ja, auch heuer gibt es wieder eine kleine Überraschung. Aber alles können wir ja nicht vorab verraten ...

Hat sich das Spektrum der Erkrankungen und Problemstellungen, mit denen sich Pädiater befassen müssen, im Laufe letzten 20 Jahre geändert? Und wirkt sich das auch auf die Themengestaltung des Pädiatrischen Frühlings aus?

Müller: Ja, natürlich hat sich in den letzten 20 Jahren Vieles geändert. Denken Sie z.B. nur an das Problem der (sogenannten) sozialen Medien - das gab es vor 20 Jahren überhaupt noch nicht. Adipositas und "Lifestyle" sind weitere Beispiele, die natürlich Einfluss nehmen auf die Programmgestaltung. Gleichzeitig haben sich aber auch alle anderen pädiatrischen Gebiete weiter entwickelt und erfordern regelmäBige „Updates". Unser Bemühen ist daher, auf neue Entwicklungen einzugehen, aber auch Altbewährtes nicht vergessen zu lassen. Dabei ist uns - wie schon erwähnt - der Pra- xisbezug besonders wichtig. Der Pädiatrische Frühling transfomiert wenn Sie so wollen - neue wissenschaftliche Erkenntnisse in praktische Anwendbarkeit.

Wird es 2020 eine Jubiläumstagung geben, und gibt es dazu schon Details, die Sie uns verraten möchten?

Müller: Das bewährte Format möchten wir an sich auch im Jubiläumsjahr 2020 bewahren. Aber natürlich wird es das eine oder andere "Highlight" geben und wohl auch eine kleine Jubiläumsfeier. Auf ein Feuerwerk werden wir aber aus Umweltgründen verzichten, wir setzen vielmehr auf ein Feuerwerk der Vorträge!

Was wünschen Sie sich persönlich für die Veranstaltung?

Müller: Ich wünsche mir, dass alle Teilnehmerinnen und Teilnehmer nach zwei Tagen mit dem Gefühl weggehen, wesentlich „dazu gelernt" zu haben. Wenn sie - nach netten Abendgesprächen oder einem Frühlingsspaziergang in der Pause - das auch noch entspannt tun, ist das eigentliche Ziel erreicht.

Vielen Dank für das interessante Gespräch!

\section{Terminaviso:}

20. Pädiatrischer Frühling, 14.-16. Mai 2020

Paediatr. Paedolog. 2019 · 54:57 https://doi.org/10.1007/s00608019-0661-2

(c) Springer-Verlag GmbH Austria, ein Teil von Springer Nature 2019

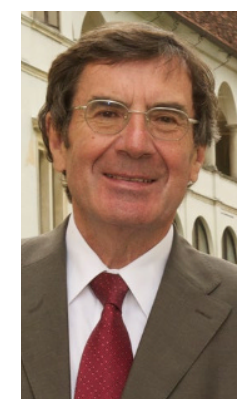

emer. Univ.-Prof. Dr. Wilhelm Müller. (৫) Univ.-Klinik für Kinder- und Jugendheilkunde Graz, Fotograf: Martin Schreiner)
Das Interview führte Dr. Renate LesskyHöhl, 21.02.2019 